\title{
On the Mechanics Underlying the Reservoir-Excess Separation in Systemic Arteries and their Implications for Pulse Wave Analysis
}

\author{
Jordi Alastruey
}

Published online: 17 December 2010

(C) The Author(s) 2010. This article is published with open access at Springerlink.com

\begin{abstract}
Several works have separated the pressure waveform $p$ in systemic arteries into reservoir $p_{r}$ and excess $p_{\text {exc }}$ components, $p=p_{r}+p_{\text {exc }}$, to improve pulse wave analysis, using windkessel models to calculate the reservoir pressure. However, the mechanics underlying this separation and the physical meaning of $p_{r}$ and $p_{\text {exc }}$ have not yet been established. They are studied here using the timedomain, inviscid and linear one-dimensional (1-D) equations of blood flow in elastic vessels. Solution of these equations in a distributed model of the 55 larger human arteries shows that $p_{r}$ calculated using a two-element windkessel model is space-independent and well approximated by the compliance-weighted space-average pressure of the arterial network. When arterial junctions are wellmatched for the propagation of forward-travelling waves, $p_{r}$ calculated using a three-element windkessel model is space-dependent in systole and early diastole and is made of all the reflected waves originated at the terminal (peripheral) reflection sites, whereas $p_{\text {exc }}$ is the sum of the rest of the waves, which are obtained by propagating the left ventricular flow ejection without any peripheral reflection. In addition, new definitions of the reservoir and excess pressures from simultaneous pressure and flow measurements at an arbitrary location are proposed here. They provide valuable information for pulse wave analysis and overcome the limitations of the current two- and threeelement windkessel models to calculate $p_{r}$.
\end{abstract}

Keywords Arterial haemodynamics - Pulse wave propagation $\cdot$ Pulse wave analysis $\cdot$ Reservoir-excess

\footnotetext{
J. Alastruey $(\bowtie)$

Departments of Bioengineering and Aeronautics,

Imperial College, London SW7 2AZ, UK

e-mail: jordi.alastruey-arimon@imperial.ac.uk
}

separation - Windkessel pressure - One-dimensional distributed model · Time-domain formulation - Conduit arteries · Well-matched bifurcations

\section{Introduction}

Several works (Wang et al. 2003; Davies et al. 2007, 2010; Aguado-Sierra et al. 2008; Tyberg et al. 2008, 2009; Parker 2009; Vermeersch et al. 2009) have suggested that the time-domain analysis of pulse wave propagation in systemic arteries is improved if the pressure waveform is separated into a reservoir pressure that varies in time, but is uniform (space-independent) throughout the arteries, and an excess pressure ${ }^{1}$ that varies in time and space due to wave propagation; the so-called reservoir-excess separation. These works have shown that the pressure waveform in systole and early diastole is made up of the combination of reservoir and excess pressures, whereas the pressure decay in late diastole is well described by the reservoir pressure only. Davies et al. (2010) have recently shown that the reservoir pressure increases with aging and correlates well with factors that determine health risk.

Despite all these studies, the physical bases of the reservoir and excess pressures have not yet been established and the reservoir-excess separation has remained an ad hoc assumption. Moreover, two different algorithms have been proposed and used to calculate the reservoir pressure (Wang et al. 2003; Aguado-Sierra et al. 2008), but their assumptions and results have not been rigorously compared.

Other separations of the pulse waveform with better understood mechanics have been suggested. Simultaneous

\footnotetext{
1 The excess pressure was called wave pressure in some papers (e.g. Tyberg et al. (2009)).
} 
pressure and velocity measurements at an arbitrary arterial location and an estimation of the local pulse wave speed allow us to calculate the forward-travelling waveform propagated from proximal locations and the backwardtravelling waveform propagated from distal locations (Westerhof et al. 1972; Parker and Jones 1990; Hughes and Parker 2009; Zhang and Li 2009). The pulse waveform simulated at an arbitrary location using the one-dimensional (1-D) formulation can also be separated into a peripheral waveform made of waves initially reflected at peripheral branches and a conduit waveform made of the remaining reflected and transmitted waves at the arterial junctions, aortic valve (when shut) and any other change in geometry and elasticity within the arterial segments (Alastruey et al. 2009). This separation showed that most of the pressure waveform consists of peripheral reflections, especially in diastole when the conduit pressure vanishes. The rest of reflected waves mainly contribute to the pressure waveform in systole and early diastole.

The purpose of this work is to theoretically and numerically study the mechanics underlying the reservoir-excess separation in systemic arteries, explore their implications for pulse wave analysis when the algorithms described in Wang et al. (2003) and Aguado-Sierra et al. (2008) are used to calculate the reservoir pressure, and find new definitions of the reservoir and excess pressures that provide valuable information for pulse wave analysis and overcome the limitations of the current algorithms. First, the algorithms used in Wang et al. (2003) and Aguado-Sierra et al. (2008) are described and compared, and their limitations are discussed ("Reservoir-Excess Separation"). These algorithms are then related to the 1-D equations of blood flow in elastic vessels ("The 1-D Formulation, From the 1-D Equations to the Windkessel Pressure, 3-Element Windkessel and 1-D Model Pressures, and Diastolic flow"), which are a reasonable approach to model pulse wave propagation in systemic arteries (Olufsen et al. 2000; Čanić and Kim 2003; Steele et al. 2003; Quarteroni and Formaggia 2004; Matthys et al. 2007). The new reservoir and excess pressures are defined in "New Reservoir and Excess Pressures". Pulse waveforms generated using the 1-D formulation in a single-vessel aortic model and a 55-segment arterial model ("Numerical Experiments") will be used to illustrate the results.

\section{Methodology}

\section{Reservoir-Excess Separation}

The reservoir-excess separation was introduced by Wang et al. (2003) using Frank's windkessel pressure $p_{w}(t)$ as the reservoir pressure, which satisfies the mass conservation (Frank 1899)
$q_{I N}=C_{T} \frac{\mathrm{dp}_{\mathrm{w}}}{\mathrm{d} t}+\frac{p_{w}-P_{\text {out }}}{R_{T}}$,

where $t$ is the time, $q_{I N}(t)$ the left ventricular flow ejection, $C_{T}$ the net compliance of the whole arterial tree, $R_{T}$ the net resistance of the peripheral systemic circulation, and $P_{\text {out }}$ the pressure at which flow to the periphery ceases. $C_{T}, R_{T}$ and $P_{\text {out }}$ were assumed to be constant and estimated from in vivo pressure and flow waveforms measured at the ascending aorta, so that Eq. 1 could be solved for $p_{w}$,

$\begin{aligned} p_{w}= & P_{\text {out }}+\left(p_{w}\left(T_{0}\right)-P_{\text {out }}\right) e^{\frac{-\left(t-T_{0}\right)}{R_{T} C_{T}}} \\ & +\frac{1}{C_{T}} e^{\frac{-t}{R_{T} C_{T}}} \int_{T_{0}}^{t} q_{I N}\left(t^{\prime}\right) e^{\frac{t^{\prime}}{R_{T} C_{T}}} d t^{\prime}, \quad t \geq T_{0},\end{aligned}$

with $p_{w}\left(T_{0}\right)$ the windkessel pressure at $t=T_{0}$. The excess pressure was defined as $p-p_{w}$, with $p$ the measured pressure, and was found to be similar to $Z_{A o} q_{I N}$ in normal conditions (Wang et al. 2003), with $Z_{A o}$ the characteristic impedance of the ascending aorta (defined as the pressureto-flow ratio of a forward-travelling wave (Milnor 1989)).

If the flow is periodic with period $T$, Eq. 1 leads to

$\overline{p_{w}}=P_{\text {out }}+R_{T} \overline{q_{I N}}$,

where $\overline{(\cdot)}=\frac{1}{T} \int_{T_{0}}^{T_{f}}(\cdot) d t$ is the mean over the time interval $\left[T_{0}, T_{f}\right]$, with $T=T_{f}-T_{0} \cdot \overline{q_{I N}}$ is, therefore, the cardiac output.

To calculate $p_{w}$ we require $q_{I N}$, which cannot always be measured in vivo. To overcome this limitation, AguadoSierra et al. (2008) proposed to calculate the reservoir pressure as the pressure $p_{r}$ satisfying the three-element windkessel equation

$\frac{p-P_{\text {out }}}{\lambda}=C_{T} \frac{\mathrm{dp}_{\mathrm{r}}}{\mathrm{d} t}+\frac{p_{r}-P_{\text {out }}}{\hat{R}}, \quad \frac{1}{\hat{R}}=\frac{1}{R_{T}}+\frac{1}{\lambda}$,

where $p$ is the measured pressure at an arbitrary location and $\lambda$ is a resistance relating $q_{I N}$ and the excess pressure $p_{\text {exc }}=p-p_{r}$; i.e.

$p_{\text {exc }}=\lambda q_{I N}, \quad p_{r}=p-\lambda q_{I N}$,

with the feet of the $q_{I N}$ and $p$ waves aligned in time to eliminate the delay between them. The constants $\lambda, P_{\text {out }}, C_{T}$ and $\hat{R}$ were determined using an iterative fitting algorithm, which means that $\lambda$, and hence $p_{r}$, were not defined explicitly.

It is important to remark that if $\lambda=Z_{A o}$, then Eq. 4 corresponds to the three-element windkessel model (Westerhof et al. 1969, 1971). Moreover, Eqs. 1 and 4 and their analogous electrical circuits (Fig. 1) show that $p_{w}$ and $p_{r}$ are conceptually different.

From Eq. 5 we have

$\overline{p_{e x c}}=\lambda \overline{q_{I N}}, \quad \overline{p_{r}}=\bar{p}-\lambda \overline{q_{I N}}$, 


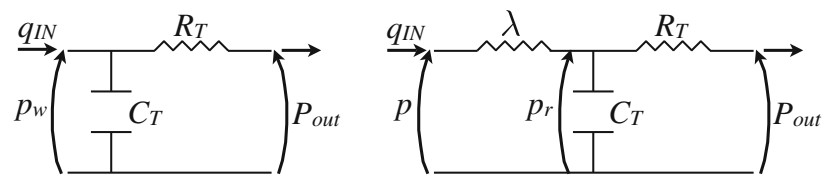

Fig. 1 Analogous electrical circuit diagrams governed by the (left) two-element windkessel Eq. 1 and (right) three-element windkessel Eq. 4

where $\overline{p_{\text {exc }}}, \overline{p_{r}}$ and $\bar{p}$ are the mean values of $p_{\text {exc }}, p_{r}$ and $p$, respectively.

\section{The 1-D Formulation}

The nonlinear terms in the 1-D formulation will be neglected since it makes the analysis simpler and it is consistent with Eqs. 1 and 4 being linear. Moreover, the effect of nonlinearities is small for the physiological parameters used (Matthys et al. 2007).

Consider the arterial system to be a network of $N$ elastic and homogeneous arterial segments (or edges) from 1 to $\mathrm{N}$, in which pulse wave propagation is modelled using the linearised 1-D equations (Alastruey et al. 2009)

$$
\left\{\begin{array}{l}
C_{1 D}^{i} \frac{\partial p^{i}}{\partial t}+\frac{\partial q^{i}}{\partial x}=0, \\
L_{1 D}^{i} \frac{\partial q^{i}}{\partial t}+\frac{\partial p^{i}}{\partial x}=-R_{1 D}^{i} q^{i}, \quad i=1, \ldots, N, \\
p^{i}=\frac{a^{i}}{C_{1 D}^{i}},
\end{array}\right.
$$

where the superscript $i$ indicates the number of the edge, $x \in\left[0, l^{i}\right]$ is the axial coordinate along the vessel, $l^{i}$ the edge length, $p^{i}(x, t)$ the average internal pressure over the luminal cross section, $q^{i}(x, t)$ the luminal flow rate, $a^{i}(x, t)+A_{0}^{i}$ the cross-sectional area of the lumen, and $A_{0}^{i}$ the area at $p^{i}=0$ assumed to be uniform within each edge. The constants

$\begin{aligned} R_{1 D}^{i} & =\frac{22 \pi \mu}{\left(A_{0}^{i}\right)^{2}}, \quad L_{1 D}^{i}=\frac{\rho}{A_{0}^{i}}, \quad C_{1 D}^{i}=\frac{3\left(A_{0}^{i}\right)^{3 / 2}}{2 E^{i} h^{i} \sqrt{\pi}}, \\ i & =1, \ldots, N,\end{aligned}$

are the viscous resistance to flow, blood inertia and elastic wall compliance, respectively, per unit length of vessel, $\rho$ is the constant mass density of blood, and $\mu$ the constant blood viscosity. The arterial wall is assumed to be thin, homogeneous, incompressible and elastic, and to deform axisymmetrically, each cross-section independent of the others. The constants $h^{i}$ and $E^{i}$ are the thickness and Young's modulus of the wall, respectively.

The equations in 7 form a system of hyperbolic partial differential equations. The characteristic variables $w_{f}^{i}$ and $w_{b}^{i}$ of this system are $w_{f, b}^{i}=q^{i} \pm \frac{p^{i}}{Z_{0}^{i}}, \quad i=1, \ldots, N$,

where $Z_{0}^{i}=\rho c^{i} / A_{0}^{i}$ is the characteristic impedance of the edge and $c^{i}=\sqrt{1 / L_{1 D}^{i} C_{1 D}^{i}}$ its pulse wave speed. Changes in pressure and flow within any edge are propagated forward by $w_{f}^{i}$ and backward by $w_{b}^{i}$ along the characteristic lines $\mathrm{d} \widetilde{x}_{f, b}^{i} / \mathrm{d} t= \pm c^{i}$, respectively, where $\widetilde{x}_{f, b}^{i}(t)$ represent lines in the $(x, t)$ space. If viscous dissipation is neglected (i.e. $\left.R_{1 D}^{i}=0, i=1, \ldots, N\right), w_{f}^{i}$ and $w_{b}^{i}, i=1, \ldots, N$, are invariant along the characteristic lines and, hence, propagate changes in pressure and flow without wave dissipation.

For convenience, the edges are indexed so that $i=1$ refers to the ascending aorta and $i=2$ to $M(M<N)$ refer to terminal edges. The inlet flow, $q^{1}(0, t)$, is assumed to be given and equal to the flow waveform from the left ventricle $q_{I N}$. The distal ends of edges numbered from 2 to $M$ are coupled to a matched RCR windkessel model relating $p_{\text {out }}^{j}(t)=p^{j}\left(l^{j}, t\right)$ to $q_{\text {out }}^{j}(t)=q^{j}\left(l^{j}, t\right), j=2, \ldots, M$, through (Alastruey et al. 2008)

$q_{\text {out }}^{j}\left(1+\frac{Z_{0}^{j}}{R_{j}}\right)+C_{j} Z_{0}^{j} \frac{\mathrm{dq}_{\text {out }}^{\mathrm{j}}}{\mathrm{d} t}=\frac{p_{\text {out }}^{j}-P_{\text {out }}}{R_{j}}+C_{j} \frac{\mathrm{dp} \mathrm{put}^{\mathrm{j}}}{\mathrm{d} t}$,

$j=2, \ldots, M$,

where $R_{j}$ and $C_{j}$ are the constant peripheral resistance and compliance, respectively.

At the junctions (or nodes) of the network, conservation of mass and continuity of pressure are enforced. For each of the three edges $a, b$ and $c$ connected at a junction, the reflection coefficients $\Gamma^{j}(j=a, b, c)$ are defined as the ratio of the change of pressure across the reflected wave to the change of pressure in the incident wave. They can be expressed as a function of the characteristic admittance of the edge $Y_{0}^{j}=1 / Z_{0}^{j}, j=a, b, c$ (Alastruey et al. 2009),

$$
\begin{aligned}
\Gamma^{a} & =\frac{Y_{0}^{a}-Y_{0}^{b}-Y_{0}^{c}}{Y_{0}^{a}+Y_{0}^{b}+Y_{0}^{c}}, \quad \Gamma^{b}=\frac{Y_{0}^{b}-Y_{0}^{c}-Y_{0}^{a}}{Y_{0}^{b}+Y_{0}^{c}+Y_{0}^{a}}, \\
\Gamma^{c} & =\frac{Y_{0}^{c}-Y_{0}^{a}-Y_{0}^{b}}{Y_{0}^{c}+Y_{0}^{a}+Y_{0}^{b}} .
\end{aligned}
$$

\section{Forward-and backward-travelling waveforms}

We can calculate the forward $\left(p_{f}, q_{f}\right)$ and backward $\left(p_{b}, q_{b}\right)$ contributions to $p$ and $q$ at an arbitrary location in the network as $p_{f, b}=\frac{1}{2}\left(p \pm Z_{0} q\right)$ and $q_{f, b}=\frac{1}{2}\left(q \pm \frac{p}{Z_{0}}\right)$ (Parker and Jones 1990). They satisfy $p_{f}=Z_{0} q_{f}$ and $p_{b}=-Z_{0} q_{b}$. The former yields $p^{i}=Z_{0}^{i} q^{i}$ when $w_{b}^{i}=0$ in Eq. 9 (i.e. backward-travelling waves are absent) and the latter $p^{i}=-Z_{0}^{i} q^{i}$ when $w_{f}^{i}=0$ (i.e. forward-travelling waves are absent) $(i=1, \ldots, N)$. 


\section{Conduit and Peripheral Pressures}

In a linear 1-D model simulation, the conduit contribution to pressure $p_{c o n}$ is obtained at any location in the network by enforcing terminal boundary conditions that completely absorb any incident wave; i.e. single resistances with $R_{j}=Z_{0}^{j}, j=2, \ldots, M$. The peripheral contribution to pressure $p_{p e r}$ is then calculated for the linear case as $p_{p e r}=$ $p-p_{\text {con }}$ (Alastruey et al. 2009).

From the 1-D Equations to the Windkessel Pressure

Integrating the first two equations in (7) over the edge length $l^{i}$ yields

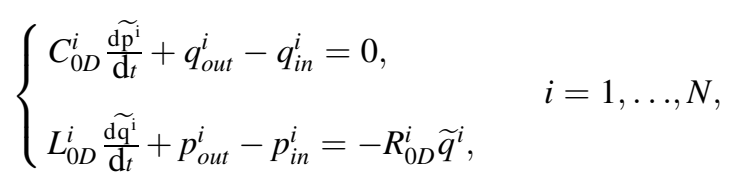

where $q_{\text {in }}^{i}(t)=q^{i}(0, t), q_{\text {out }}^{i}(t)=q^{i}\left(l^{i}, t\right), p_{\text {in }}^{i}(t)=p^{i}(0, t)$, $p_{\text {out }}^{i}(t)=p^{i}\left(l^{i}, t\right), R_{0 D}^{i}=R_{1 D}^{i} l^{i}, L_{0 D}^{i}=L_{1 D}^{i} l^{i}, C_{0 D}^{i}=C_{1 D}^{i} l^{i}$, and $\widetilde{p}^{i}(t)=\frac{1}{l^{i}} \int_{0}^{l^{i}} p^{i} \mathrm{~d} x$ and $\widetilde{q}^{i}(t)=\frac{1}{l^{i}} \int_{0}^{l^{i}} q^{i} \mathrm{~d} x$ are the spaceaveraged pressure and flow over an edge of length $l^{i}$. Assuming conservation of mass at the nodes of the network and combining the $N$ equations of conservation of mass in 12 yield the following equation for the conservation of mass for the network,

$q_{I N}=C_{c} \frac{\mathrm{dp}_{\mathrm{C}}}{\mathrm{d} t}+q_{C}$,

$p_{C}=\frac{1}{C_{c}} \sum_{i=1}^{N} C_{0 D}^{i} \tilde{p}^{i}, \quad C_{c}=\sum_{i=1}^{N} C_{0 D}^{i}$,

where $p_{C}(t)$ is the compliance-weighted space-average pressure of the network, $C_{c}$ the total conduit compliance and $q_{C}(t)=\sum_{j=2}^{M} q_{\text {out }}^{j}$ the total peripheral outflow driven by $p_{C}$.

Hereafter, $R_{0 D}^{i}=0, i=1, \ldots, N$, will be assumed since the resistance in large arteries is much smaller than peripheral resistances (Caro et al. 1978) [Chap. 12]. Taking $L_{0 D}^{i}=0, i=1, \ldots, N$, leads to $c^{i}=\sqrt{1 / L_{1 D}^{i} C_{1 D}^{i}} \rightarrow \infty$, $i=1, \ldots, N$, (i.e. changes in pressure and flow occur synchronously) and reduces the $N$ equations of balance of momentum in (12) to

$p_{\text {out }}^{i}=p_{\text {in }}^{i}, \quad i=1, \ldots, N$.

Furthermore, $p_{\text {out }}^{i}=p_{\text {in }}^{i}=\widetilde{p}^{i}, i=1, \ldots, N$, at any location of the $i$-th arterial segment, since 15 holds for any length $l^{i}$.

Assuming continuity of pressure at the nodes of the network, we obtain $\widetilde{p}^{i}=\widetilde{p}_{w}, i=1, \ldots, N$, where $\widetilde{p}_{w}(t)$ is the space-independent pressure to which the 1-D model pressures in 7 reduce when $R_{0 D}^{i}=0$ and $L_{0 D}^{i}=0$, $i=1, \ldots, N$. Equation 13 then becomes

$$
\begin{aligned}
& q_{I N}=C_{c} \frac{\mathrm{d} \widetilde{\mathrm{p}}_{\mathrm{w}}}{\mathrm{d} t}+\widetilde{q}_{w}, \\
& \widetilde{q}_{w}=C_{p} \frac{\mathrm{d} \widetilde{\mathrm{p}}_{\mathrm{w}}}{\mathrm{d} t}+\frac{\widetilde{p}_{w}-P_{\text {out }}}{R_{T}}-\sum_{j=2}^{M} \frac{C_{j} Z_{0}^{j} R_{j}}{R_{j}+Z_{0}^{j}} \frac{\mathrm{dq}_{\text {out }}^{\mathrm{j}}}{\mathrm{d} t}, \\
& \frac{1}{R_{T}}=\sum_{j=2}^{M} \frac{1}{R_{j}+Z_{0}^{j}}, \quad C_{p}=\sum_{j=2}^{M} \frac{R_{j} C_{j}}{R_{j}+Z_{0}^{j}},
\end{aligned}
$$

where $\widetilde{q}_{w}(t)$ is the total outflow to the periphery driven by $\widetilde{p}_{w}$ and $C_{p}$ is the total peripheral compliance. The solution to Eq. 16 is

$$
\begin{aligned}
\tilde{p}_{w}= & P_{\text {out }}+\left(\widetilde{p}_{w}\left(T_{0}\right)-P_{\text {out }}\right) e^{\frac{-\left(t-T_{0}\right)}{R_{T} C_{T}}} \\
& +\frac{e^{\frac{-t}{R_{T} C_{T}}}}{C_{T}} \int_{T_{0}}^{t}\left(q_{\text {IN }}\left(t^{\prime}\right)+\sum_{j=2}^{M} \frac{C_{j} Z_{0}^{j} R_{j}}{R_{j}+Z_{0}^{j}} \frac{\mathrm{dq}_{\text {out }}^{j}\left(\mathrm{~d} t^{\prime}\right)}{\mathrm{d} t^{\prime}}\right) \\
& \times e^{\frac{d t^{\prime}}{R_{T} C_{T}}} d t^{\prime}, \quad t \geq T_{0},
\end{aligned}
$$

where $\widetilde{p}_{w}\left(T_{0}\right)$ is the pressure $\widetilde{p}_{w}$ at $t=T_{0}$ and $C_{T}=C_{c}+C_{p}$ is the total compliance. Finally, Eq. 16 reduces to Frank's windkessel Eq. 1, with $\widetilde{p}_{w}=p_{w}$, when $C_{j}=0, j=2, \ldots, M$; i.e. we only have conduit compliance $C_{c}$.

Next, the implications of time-averaging Eqs. 13, 14 and 16 will be explored.

Time-averaged behaviour. Integrating Eq. 13 over the interval $\left[T_{0}, T_{f}\right]$ yields

$T\left(\overline{q_{I N}}-\sum_{j=2}^{M} \overline{q_{\text {out }}^{j}}\right)=\sum_{i=1}^{N} C_{0 D}^{i}\left[\widetilde{p}^{i}\left(T_{f}\right)-\widetilde{p}^{i}\left(T_{0}\right)\right]$.

If the flow is periodic with a period $T$, then Eqs. 10 and 18 lead to

$\overline{q_{I N}}=\sum_{j=2}^{M} \overline{q_{\text {out }}^{j}}=\sum_{j=2}^{M} \frac{\overline{p_{\text {out }}^{j}}-P_{\text {out }}}{R_{j}+Z_{0}^{j}}$.

Integrating the balance of momentum in 12 over the interval $\left[T_{0}, T_{f}\right]$ and assuming periodic flow with a period $T$ yields $\overline{p_{\text {out }}^{i}}=\overline{p_{\text {in }}^{i}}, i=1, \ldots, N$. Since Eq. 12 holds for any length $l^{i}$, we have $\overline{p_{\text {out }}^{i}}=\overline{p_{\text {in }}^{i}}=\overline{p^{i}}, i=1, \ldots, N ; p^{i}$ being evaluated at any location of the $i$-th arterial segment. Combining these results with continuity of pressure at the nodes of the network leads to $\overline{p_{\text {out }}^{j}}=\overline{p^{i}}, j=2, \ldots, M$ and $i=1, \ldots, N$. Equation 19, hence, reduces to

$\overline{p^{i}}=P_{\text {out }}+R_{T} \overline{q_{I N}}, \quad i=1, \ldots, N ;$

i.e. the mean pressure at any location in the network is space-independent.

Combining Eqs. 14 and 20 yields 
$\overline{p_{C}}=P_{\text {out }}+R_{T} \overline{q_{I N}}$,

and integrating Eq. 16 over the interval $\left[T_{0}, T_{f}\right]$ yields

$\overline{\widetilde{p}_{w}}=P_{\text {out }}+R_{T} \overline{q_{I N}}$.

Thus, $\overline{\widetilde{p}_{w}}=\overline{p_{C}}=\overline{p^{i}}, i=1, \ldots, N$.

\section{3-Element Windkessel and 1-D Model Pressures}

The calculation of the reservoir pressure using Eq. 4 is based on the assumptions of well-matched arterial junctions for the transmission of forward-travelling waves (i.e. $\Gamma=0$ at the outlets of internal edges) and $q_{I N}$ being proportional to $p_{\text {exc }}$ except for a time shift to account for the propagation of $q_{I N}$ to the measurement site of $p$ (Aguado-Sierra et al. 2008). The latter is satisfied if the outlets of terminal edges are completely absorbent (i.e. they are single resistances with $\left.R_{j}=Z_{0}^{j}, j=2, \ldots, M\right)$, so that reflected waves are absent in the system (i.e. $w_{b}^{i}=0, i=1, \ldots, N$ ). Under these assumptions, the pressure $Z_{A o} q_{I N}$ at the aortic root will maintain its shape throughout the network, leading to $Z_{\text {Ao }} q_{I N}=Z_{0}^{i} q^{i}, i=1, \ldots, N$ with $q_{I N}$ and $q^{i}$ time-aligned (so that the feet of both waves coincide), and $p_{r}^{i}=0, i=1, \ldots, N$, if $\lambda=Z_{\text {Ao }}$ (Eq. 5).

If $p_{\text {exc }}^{i}$ is calculated using Eq. 5 with $\lambda=Z_{A o}$ in a network with reflective terminal boundary conditions (but still well-matched junctions for forward-travelling waves), then $p_{\text {exc }}^{i}=Z_{A o} q_{I N}=p_{c o n}^{i}$ and $p_{r}^{i}=p_{p e r}^{i}(i=1, \ldots, N)$; i.e. $p_{\text {exc }}^{i}$ is the part of $p^{i}$ due to waves travelling from the aortic root to the periphery and $p_{r}^{i}$ is the part of $p^{i}$ made of all the reflected waves originated at the terminal (peripheral) reflection sites.

\section{Diastolic Flow}

In normal diastolic conditions $q_{I N}=0$, so that Eqs. 13 and 16 reduce to

$q_{C}=-\sum_{i=1}^{N} C_{0 D}^{i} \frac{\mathrm{d} \widetilde{\mathrm{p}}^{\mathrm{i}}}{\mathrm{d} t}=-C_{c} \frac{\mathrm{dp} \mathrm{p}_{\mathrm{C}}}{\mathrm{d} t}, \quad \widetilde{q}_{w}=-C_{c} \frac{\mathrm{d} \widetilde{\mathrm{p}}_{\mathrm{w}}}{\mathrm{d} t}$.

Alastruey et al. (2009) showed that $p^{i}, i=1, \ldots, N$, converge to $\widetilde{p}_{w}(t)$ (Eq. 17) in diastole. According to the analysis in "From the 1-D Equations to the Windkessel Pressure", this implies that $L_{0 D}^{i} \rightarrow 0, i=1, \ldots, N$ in diastole and, hence, $c^{i}=\sqrt{1 / L_{1 D}^{i} C_{1 D}^{i}} \rightarrow \infty, i=1, \ldots, N$; i.e. changes in pressure and flow occur synchronously. The convergence of $p^{i}, i=1, \ldots, N$, to $\widetilde{p}_{w}$ leads to $\widetilde{p}_{w}=p_{C}$ and, hence, $\widetilde{q}_{w}=q_{C}$, according to Eq. 23.

This equation also shows that the contribution of each edge to the total diastolic outflow $q_{C}$ increases with the edge compliance $C_{0 D}^{i}$. Moreover, the conservation of mass in Eq. 7 with $p^{i}=\widetilde{p}_{w}, i=1, \ldots, N$, shows that the flow in each edge is linearly dependent on $x$,

$q^{i}=q_{i n}^{i}-C_{1 D}^{i} \frac{\mathrm{d} \widetilde{\mathrm{p}}_{\mathrm{w}}}{\mathrm{d} t} x, \quad i=1, \ldots, N$.

According to Eq. 17, $\widetilde{p}_{w}$ decays exponentially when $C_{j}=0, j=2, \ldots, M$. In this case,

$\tilde{p}_{w}=p_{w}=P_{\text {out }}+\left(p_{w}\left(T_{d}\right)-P_{\text {out }}\right) e^{\frac{-\left(t-T_{d}\right)}{R_{T} C_{T}}}, \quad t \geq T_{d}$,

where $p_{w}\left(T_{d}\right)$ is the windkessel pressure at the beginning of diastole, $t=T_{d}$.

\section{New Reservoir and Excess Pressures}

This work will explore the implications of defining the reservoir and excess pressures from simultaneous $p$ and $q$ measurements at an arbitrary location as

$\widetilde{p_{r}}=p-Z_{0} q, \quad \widetilde{p_{\text {exc }}}=Z_{0} q$.

A tilde is used to distinguish these new reservoir and excess pressures from the corresponding pressures calculated using the three-element windkessel model $\left(p_{r}\right.$ and $p_{\text {exc }}$ ). Note that $\widetilde{p_{r}}=p_{r}$ at the aortic root if $\lambda=Z_{A o}$. Using $p=p_{f}+p_{b}$ and $q=q_{f}+q_{b}$, and the relations $q_{f}=$ $p_{f} / Z_{0}$ and $q_{b}=-p_{b} / Z_{0}$ shown in "Forward- and Backward-Travelling Waveforms", $\widetilde{p_{r}}$ and $\widetilde{p_{e x c}}$ satisfy

$\widetilde{p_{r}}=2 p_{b}, \quad \widetilde{p_{\text {exc }}}=p_{f}-p_{b}$

at any location in the arterial network. Thus, $\widetilde{p_{r}}$ is made of waves coming from distal locations only and $\widetilde{p_{e x c}}$ is the difference between proximal and distal contributions to $p$. In a well-matched network for forward-travelling waves with the outlets of terminal edges completely absorbent, $p_{b}=0$ and, hence, $p=p_{f}$. Therefore, $\widetilde{p_{r}}=0$ and $\widetilde{p_{\text {exc }}}=p=Z_{0} q$.

\section{Numerical Experiments}

The theoretical results described above will be illustrated using waveforms simulated in a single-vessel 1-D model of the human aorta coupled to a matched RCR windkessel model of the rest of the circulation (Alastruey et al. 2008). Two distributed models will also be used: a 55-artery 1-D model of the larger systemic arteries in the human, referred to as 'normal 55-artery model' (Fig. 2), and a version of this model with modified radii so that junctions are wellmatched $(\Gamma=0)$ for forward-travelling waves, referred to as 'well-matched 55-artery model'. Tables 1,2 and 3 show the parameters of these models, which have the same total resistance $R_{T}$ and compliance $C_{T}$, and outflow pressure $P_{\text {out }}=0$. Figure $4 \mathrm{~b}$ shows the flow rate $q_{I N}$ prescribed at the aortic root of the three models; it is periodic until $t=$ 


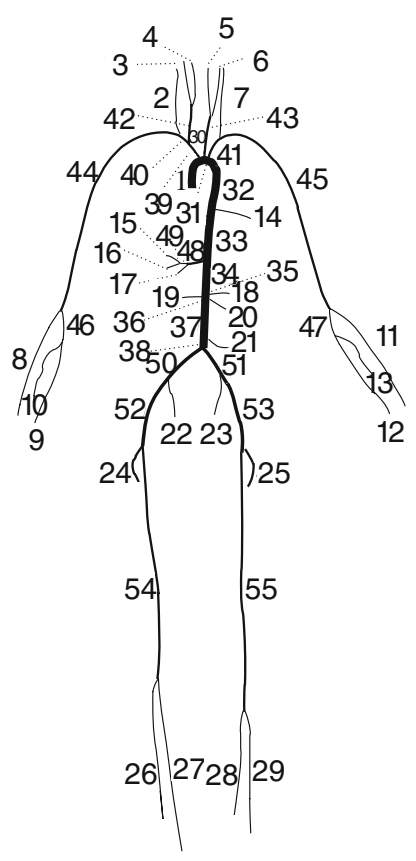

Fig. 2 Connectivity of the 55 larger systemic arteries in the human, as proposed in Stergiopulos et al. (1992). Their names and properties are shown in Tables 2 and 3

$10 \mathrm{~s}$ and zero afterward to study the relaxation of these models.

The equations in 7 were solved in each model using a discontinuous Galerkin scheme with a spectral $/ h p$ spatial discretisation, a second-order Adams-Bashforth time-integration scheme, a time step of $100 \mu \mathrm{s}$, and zero pressures and flows as initial conditions (Alastruey 2006). Arterial edges were divided in non-overlaping elements with a $2 \mathrm{~cm}$ length (when physically possible) and a polynomial and quadrature order of 3. Elements or edges shorter than $2 \mathrm{~cm}$ were given a polynomial and quadrature order of 2 .

\section{Results}

\section{Single-Vessel Aorta}

Blood pressure $p$ at any location in the single-vessel aortic model and the reservoir $p_{r}$, compliance-weighted spaceaverage $p_{C}$ and windkessel $\widetilde{p}_{w}$ pressures, calculated using Eqs. 5 (with $\lambda=Z_{A o}$ ), 14 and 17 (with $T_{0}=0$ and $\widetilde{p}_{w}\left(T_{0}\right)=0$ to satisfy the initial conditions of the 1-D model), respectively, tend to the same shape with the increasing time in diastole. Figure $3 \mathrm{a}, \mathrm{b}$ show these pressures ( $p$ and $p_{r}$ in the midpoint) once the flow has become periodic. Figure $3 \mathrm{a}$ also shows the excess pressure $p_{\text {exc }}$ in the midpoint, which is proportional to $q_{I N}$. The difference between $\widetilde{p}_{w}$ and $p_{C}$ is smaller than $2 \mathrm{kPa}$ in systole and becomes smaller with time in diastole (Fig. 3b). From 9 to
Table 1 Parameters of the single-vessel model of the human aorta based on Caro et al. (1978) and Westerhof et al. (1969)

\begin{tabular}{ll}
\hline Parameter & Value \\
\hline Length $l$ & $0.4 \mathrm{~m}$ \\
Cross-sectional area $A_{0}$ & $\pi \mathrm{cm}^{2}$ \\
Wall thickness $h$ & $1.0 \mathrm{~mm}$ \\
Blood mass density $\rho$ & $1050 \mathrm{Kg} \mathrm{m}^{-3}$ \\
Elastic Young's modulus $E$ & $0.4 \mathrm{MPa}^{-1}$ \\
Peripheral compliance $C$ & $8.2 \mathrm{~m}^{3} \mathrm{GPa}^{-1}$ \\
Peripheral resistance $R_{T}$ & $134.2 \mathrm{MPa} \mathrm{s} \mathrm{m}^{-3}$ \\
\hline
\end{tabular}

The resulting pulse wave speed is $c=5.0 \mathrm{~m} \mathrm{~s}^{-1}$

$10 \mathrm{~s}$, the mean values of $p_{r}+p_{e x c}$ and $p$ at any location, $p_{C}$ and $\widetilde{p}_{w}$ are $12.5 \mathrm{kPa}$; the same value as predicted by Eqs. 6 , 20, 21 and 22.

Figure $3 \mathrm{c}$ shows that $p_{r}$ calculated with $\lambda=Z_{A o}$ changes in space in systole and early diastole. At any location, $p_{r}$ is identical to $p_{p e r}$ and $p_{e x c}$ is identical to $p_{c o n}$. On the other hand, $\widetilde{p_{r}}$ is space-independent except for the time alignment (Fig. 3d), and $\widetilde{p_{e x c}}$ is proportional to the flow at any location (Fig. 4a). At the inlet, $\widetilde{p_{r}}=p_{r}$ and $\widetilde{p_{\text {exc }}}=p_{\text {exc }}$.

Figure $4 \mathrm{~b}$ shows the blood flow $q$ in the midpoint of the vessel and the convergence of the outflows $q_{C}$ and $\widetilde{q}_{w}$ (calculated using Eqs. 13 and 16) to the same shape in late diastole. From 9 to $10 \mathrm{~s}$, the mean values of $q$ at any location, $q_{C}$ and $\widetilde{q}_{w}$ are equal to $\overline{q_{I N}}=92.9 \mathrm{ml} \mathrm{s}^{-1}$. During approximately the last half of diastole the flow satisfies $q=-C_{1 D} \frac{\widetilde{\mathrm{d}}_{\mathrm{w}}}{\mathrm{d} t} x$ (Eq. 24); i.e. $q$ increases linearly from zero at the inlet to the exponential shape dictated by $q_{C}=\widetilde{q}_{w}$ at the outlet.

The forward and backward parts of $p$ and $q$ are spaceindependent except for the time alignment. Figure $5 \mathrm{a}, \mathrm{b}$ show $p_{f}, p_{b}, q_{f}$ and $q_{b}$ in the midpoint: in most of the cardiac cycle $p_{f}$ is greater than $p_{b}$ and $q_{f}$ is greater than $-q_{b}$; in most of diastole $p_{f}$ and $p_{b}$ have a similar shape, as do $q_{f}$ and $-q_{b}$. At the inlet, $p_{f}=p_{b}$ and $q_{f}=-q_{b}$ when $q_{I N}=0$.

Figure 5c shows $\widetilde{p_{r}}$ and $\widetilde{p_{e x c}}$ in the midpoint; $\widetilde{p_{r}}$ is the major component of $p$, especially in diastole, and $\widetilde{p_{e x c}}$ significantly determines the pressure shape in systole and early diastole. During approximately the last half of diastole $\widetilde{p_{e x c}}=Z_{0} q=-Z_{0} C_{1 D} \frac{\widetilde{\mathrm{dp}_{\mathrm{w}}}}{\mathrm{d} t} x$, in agreement with Eqs. 24 and 26. Thus, $\widetilde{p_{\text {exc }}}=0$ at the inlet so that $\widetilde{p_{r}}$ captures all the diastolic decay. As we approach the outlet $\widetilde{p_{\text {exc }}}$ increases and $\widetilde{p_{r}}$ decreases.

\section{5-Artery Network}

In diastole, pressures everywhere in the normal and wellmatched 55-artery models eventually converge to $\widetilde{p}_{w}$ 
Table 2 Length, initial radius and wave speed of each arterial edge in the 55-artery network in Fig. 2

\begin{tabular}{|c|c|c|c|c|c|}
\hline Arterial edge & Length $(\mathrm{cm})$ & Radius (mm) & $c\left(\mathrm{~m} \mathrm{~s}^{-1}\right)$ & $\Gamma$ inlet & $\Gamma$ outlet \\
\hline 1. Ascending aorta & 4.0 & $14.5(14.7)$ & $4.0(6.2)$ & - & 0.1 \\
\hline 2. R. vertebral & 14.8 & $1.9(2.4)$ & $8.3(11.3)$ & -0.9 & - \\
\hline 3. R. external carotid & 17.7 & $1.3(3.8)$ & $9.6(8.7)$ & -0.9 & - \\
\hline 4. R. internal carotid & 17.7 & $1.3(3.8)$ & $9.9(9.0)$ & -0.9 & - \\
\hline 5. L. internal carotid & 17.7 & $1.3(3.4)$ & $9.9(9.6)$ & -0.9 & - \\
\hline 6. L. external carotid & 17.7 & $1.3(3.4)$ & $9.6(9.2)$ & -0.9 & - \\
\hline 7. L. vertebral & 14.8 & $1.9(2.0)$ & $8.3(12.5)$ & -0.9 & - \\
\hline 8. R. radial & 23.5 & $1.6(3.7)$ & $8.8(9.0)$ & -0.8 & - \\
\hline 9. R. ulnar II & 17.1 & $1.9(4.3)$ & $8.2(8.5)$ & -0.2 & - \\
\hline 10. R. interosseous & 7.9 & $0.9(1.9)$ & $13.2(14.1)$ & -0.9 & - \\
\hline 11. L. radial & 23.5 & $1.6(3.2)$ & $8.8(9.5)$ & -0.8 & - \\
\hline 12. L. ulnar II & 17.1 & $1.9(3.8)$ & $8.2(9.1)$ & -0.2 & - \\
\hline 13. L. interosseous & 7.9 & $0.9(1.7)$ & $13.2(14.9)$ & -0.9 & - \\
\hline 14. Intercostals & 8.0 & $1.8(3.1)$ & $6.3(7.3)$ & -1.0 & - \\
\hline 15. Gastric & 7.1 & $1.8(2.2)$ & $6.0(8.3)$ & -0.6 & - \\
\hline 16. Splenic & 6.3 & $2.8(3.4)$ & $5.3(7.3)$ & 0.1 & - \\
\hline 17. Hepatic & 6.6 & $2.2(4.6)$ & $5.6(6.1)$ & -0.6 & - \\
\hline 18. Superior mesenteric & 5.9 & $4.4(4.1)$ & $4.8(7.6)$ & -0.6 & - \\
\hline 19. R. renal & 3.2 & $2.6(3.5)$ & $5.4(7.2)$ & -0.8 & - \\
\hline 20. L. renal & 3.2 & $2.6(3.5)$ & $5.4(7.2)$ & -0.9 & - \\
\hline 21. Inferior mesenteric & 5.0 & $1.6(2.5)$ & $6.2(7.6)$ & -0.9 & - \\
\hline 22. R. internal iliac & 5.0 & $2.0(4.1)$ & $10.7(11.6)$ & -0.8 & - \\
\hline 23. L. internal iliac & 5.0 & $2.0(4.1)$ & $10.7(11.6)$ & -0.8 & - \\
\hline 24. R. deep femoral & 12.6 & $2.2(3.6)$ & $7.8(9.5)$ & -0.5 & - \\
\hline 25. L. deep femoral & 12.6 & $2.2(3.6)$ & $7.8(9.5)$ & -0.5 & - \\
\hline 26. R. anterior tibial & 34.3 & $1.3(2.0)$ & $13.1(16.4)$ & -0.8 & - \\
\hline 27. R. posterior tibial & 32.1 & $1.9(3.8)$ & $11.5(12.8)$ & -0.4 & - \\
\hline 28. L. posterior tibial & 32.1 & $1.9(3.8)$ & $11.5(12.8)$ & -0.4 & - \\
\hline 29. L. anterior tibial & 34.3 & $1.3(2.0)$ & $13.1(16.5)$ & -0.8 & - \\
\hline 30. Aortic arch I & 2.0 & $11.2(12.6)$ & $4.0(5.8)$ & -0.3 & 0.0 \\
\hline 31. Aortic arch II & 3.9 & $10.7(11.9)$ & $3.9(5.7)$ & -0.1 & 0.0 \\
\hline 32. Thoracic aorta I & 5.2 & $10.0(11.2)$ & $4.0(5.8)$ & -0.1 & 0.4 \\
\hline 33. Thoracic aorta II & 10.4 & $6.6(10.7)$ & $4.6(5.7)$ & -0.5 & -0.1 \\
\hline 34. Abdominal aorta I & 5.3 & $6.1(9.2)$ & $4.6(5.8)$ & -0.2 & -0.2 \\
\hline 35. Abdominal aorta II & 1.0 & $6.0(8.4)$ & $4.4(5.7)$ & -0.2 & -0.1 \\
\hline 36. Abdominal aorta III & 1.0 & $5.9(7.9)$ & $4.4(5.9)$ & -0.1 & 0.0 \\
\hline 37. Abdominal aorta IV & 10.6 & $5.6(7.3)$ & $4.4(5.9)$ & -0.1 & 0.0 \\
\hline 38. Abdominal aorta V & 1.0 & $5.2(6.8)$ & $4.2(5.7)$ & -0.1 & 0.1 \\
\hline 39. Brachiocephalic & 3.4 & $6.2(7.0)$ & $4.3(6.3)$ & -0.8 & 0.2 \\
\hline 40. R. subclavian & 3.4 & $4.2(5.4)$ & $4.8(6.5)$ & -0.5 & 0.2 \\
\hline 41. L. subclavian & 3.4 & $4.2(4.7)$ & $4.8(7.0)$ & -0.9 & 0.2 \\
\hline 42. R. common carotid & 17.7 & $3.7(4.7)$ & $4.9(6.8)$ & -0.6 & 0.8 \\
\hline 43. L. common carotid & 20.8 & $3.7(4.2)$ & $4.9(7.2)$ & -0.9 & 0.8 \\
\hline 44. R. brachial & 42.2 & $3.2(5.2)$ & $5.5(6.7)$ & -0.4 & 0.4 \\
\hline 45. L. brachial & 42.2 & $3.2(4.6)$ & $5.5(7.1)$ & -0.4 & 0.4 \\
\hline 46. R. ulnar I & 6.7 & $2.2(4.5)$ & $7.8(8.3)$ & -0.6 & 0.1 \\
\hline 47. L. ulnar I & 6.7 & $2.2(4.0)$ & $7.8(8.9)$ & -0.6 & 0.1 \\
\hline 48. Celiac I & 1.0 & $3.9(5.9)$ & $4.8(6.1)$ & -0.7 & 0.4 \\
\hline
\end{tabular}


Table 2 continued

\begin{tabular}{|c|c|c|c|c|c|}
\hline Arterial edge & Length $(\mathrm{cm})$ & Radius (mm) & $c\left(\mathrm{~m} \mathrm{~s}^{-1}\right)$ & $\Gamma$ inlet & $\Gamma$ outlet \\
\hline 49. Celiac II & 1.0 & $2.0(4.0)$ & $6.8(7.4)$ & -0.7 & -0.5 \\
\hline 50. R. common iliac & 5.8 & $3.6(5.1)$ & $4.9(6.3)$ & -0.5 & 0.2 \\
\hline 51. L. common iliac & 5.8 & $3.6(5.1)$ & $4.9(6.3)$ & -0.5 & 0.2 \\
\hline 52. R. external iliac & 14.4 & $3.0(4.8)$ & $7.2(8.7)$ & -0.4 & 0.0 \\
\hline 53. L. external iliac & 14.4 & $3.0(4.8)$ & $7.2(8.7)$ & -0.4 & 0.0 \\
\hline 54. R. femoral & 44.3 & $2.2(3.6)$ & $8.0(9.7)$ & -0.5 & 0.2 \\
\hline 55. L. femoral & 44.3 & $2.2(3.6)$ & $8.0(9.7)$ & -0.5 & 0.2 \\
\hline
\end{tabular}

They are based on data in Stergiopulos et al. (1992). The last two columns show the reflection coefficients at the inlet and outlet of edges connected to junctions. The radii and wave speeds in brackets yield well-matched junctions for forward-travelling waves (with $\Gamma=0.000$ at the outlets of internal edges)

Table 3 Peripheral resistances and compliances at the terminal edges of the 55-artery network in Fig. 2

\begin{tabular}{|c|c|c|}
\hline Arterial edge & $\begin{array}{l}R_{j}+Z_{0}^{j} \\
\left(10^{10} \mathrm{~Pa} \mathrm{~s} \mathrm{~m}^{-3}\right)\end{array}$ & $C_{j}\left(10^{-10} \mathrm{~m}^{3} \mathrm{~Pa}^{-1}\right)$ \\
\hline 2. R. vertebral & 0.60 & $0.93(0.84)$ \\
\hline 3. R. external carotid & 1.39 & $0.40(0.36)$ \\
\hline 4. R. internal carotid & 1.39 & $0.40(0.36)$ \\
\hline 5. L. internal carotid & 1.39 & $0.40(0.36)$ \\
\hline 6. L. external carotid & 1.39 & $0.40(0.36)$ \\
\hline 7. L. vertebral & 0.60 & $0.93(0.84)$ \\
\hline 8. R. radial & 0.53 & $1.06(0.96)$ \\
\hline 9. R. ulnar II & 0.53 & $1.06(0.96)$ \\
\hline 10. R. interosseous & 8.43 & $0.07(0.06)$ \\
\hline 11. L. radial & 0.53 & $1.06(0.96)$ \\
\hline 12. L. ulnar II & 0.53 & $1.06(0.96)$ \\
\hline 13. L. interosseous & 8.43 & $0.07(0.06)$ \\
\hline 14. Intercostals & 0.14 & $4.02(3.64)$ \\
\hline 15. Gastric & 0.54 & $1.03(0.93)$ \\
\hline 16. Splenic & 0.23 & $2.41(2.18)$ \\
\hline 17. Hepatic & 0.36 & $1.54(1.39)$ \\
\hline 18. Superior mesenteric & 0.09 & $6.00(5.43)$ \\
\hline 19. R. renal & 0.11 & $4.94(4.47)$ \\
\hline 20. L. renal & 0.11 & $4.94(4.47)$ \\
\hline 21. Inferior mesenteric & 0.69 & $0.81(0.73)$ \\
\hline 22. R. internal iliac & 0.79 & $0.70(0.64)$ \\
\hline 23. L. internal iliac & 0.79 & $0.70(0.64)$ \\
\hline 24. R. deep femoral & 0.48 & $1.17(1.06)$ \\
\hline 25. L. deep femoral & 0.48 & $1.17(1.06)$ \\
\hline 26. R. anterior tibial & 0.56 & $1.00(0.90)$ \\
\hline 27. R. posterior tibial & 0.48 & $1.17(1.06)$ \\
\hline 28. L. posterior tibial & 0.48 & $1.17(1.06)$ \\
\hline 29. L. anterior tibial & 0.56 & $1.00(0.90)$ \\
\hline
\end{tabular}

They yield $R_{T}=134.2 \mathrm{MPa} \mathrm{s} \mathrm{m}{ }^{-3}$ and $C_{T}=12.0 \mathrm{~m}^{3} \mathrm{GPa}^{-1}$. In all these edges $P_{\text {out }}=0$. The compliances in brackets correspond to the network with well-matched junctions for forward-travelling waves calculated using Eq. 17 with $T_{0}=0$ and $\widetilde{p}_{w}\left(T_{0}\right)=0$. Figure 6 shows this convergence at four locations in both models once the flow is periodic, including their decay up to $t=10.4 \mathrm{~s}$ due to $q_{I N}=0$. The diastolic pressure decay is also well captured by $p_{p e r}$ and $p_{r}$ (with $\lambda=Z_{A o}$ ) (Fig. 7). Moreover, $p_{p e r}=p_{r}$ and $p_{c o n}=p_{e x c}$ everywhere in the well-matched model (Fig. 7b). In the normal model, $p_{\text {per }}$ and $p_{r}$, and $p_{c o n}$ and $p_{\text {exc }}$ differ in systole and early diastole (Fig. 7a). Figure 8a compares $p_{p e r}=p_{r}$ at several locations in the well-matched model, showing their space dependency. Both $p_{p e r}$ and $p_{r}$ are also space-dependent in the normal model.

In both networks, $\widetilde{p_{r}}$ is equal to $p_{r}\left(\lambda=Z_{A o}\right)$ at the inlet of the ascending aorta and space-independent, except for the time alignment, within any edge. Figure $8 \mathrm{~b}$ shows this in the left brachial artery of the normal model. Comparison of $\widetilde{p_{r}}$ within several edges shows its convergence to the decay given by $\widetilde{p}_{w}$, except for the time alignment (Fig. 8c).

In systole, the difference between $\widetilde{p}_{w}$ and $p_{C}$ is less than $0.8 \mathrm{kPa}$ in the normal model (Fig. 9a) and $0.35 \mathrm{kPa}$ in the well-matched model (Fig. 9b). This difference decreases with time in diastole. Once the flow has become periodic, the mean values (over a cardiac cycle) of $p_{r}+p_{\text {exc }}$ and $p$ at any location, $p_{C}$ and $\widetilde{p}_{w}$ are $12.5 \mathrm{kPa}$; the same value as predicted by Eqs. 6, 20, 21 and 22. In both models, the outflows $q_{C}$ and $\widetilde{q}_{w}$ tend to the same shape with the increasing time in diastole (Fig. 9c,d).

Within any edge in both networks, $p_{f}, p_{b}, q_{f}$ and $q_{b}$ are space-independent except for the time alignment. Figure $10 \mathrm{a}, \mathrm{b}$ show them in the midpoint of the thoracic aorta of the normal model. In diastole $p_{f}$ is similar to $p_{b}$ in shape, and so is $q_{f}$ to $-q_{b}$. They differ more in systole, with $p_{f}$ and $q_{f}$ being dominant. Figure $10 \mathrm{c}$ shows $\widetilde{p_{r}}$ and $\widetilde{p_{e x c}}$ at the same location; $\widetilde{p_{r}}$ is the major component of the diastolic decay and $\widetilde{p_{\text {exc }}}$ is greater in systole than in diastole. This result is observed at any location in both models. At the aortic root and in diastole, $\widetilde{p_{e x c}}=0$ and $\widetilde{p_{r}}$ produces all the pressure decay. 
Fig. 3 a Pressure $p$ and reservoir $p_{r}$ and excess $p_{\text {exc }}$ pressures calculated using the three-element windkessel model with $\lambda=Z_{A o}$ in the midpoint of the single-vessel aortic model, and windkessel pressure $\widetilde{p}_{w}$ with time. $\mathbf{b} \widetilde{p}_{w}$ and compliance-weighted spaceaverage pressure $p_{C}$. $\mathbf{c}$ Reservoir pressure $p_{r}$ calculated using the three-element windkessel model with $\lambda=Z_{A o}$ at $x=0$ (inlet), $l /$ 4 (1/4), $l / 2$ (mid) and 3l/4 (3/4). d New reservoir pressure $\widetilde{p_{r}}$ at the same locations as in (c)

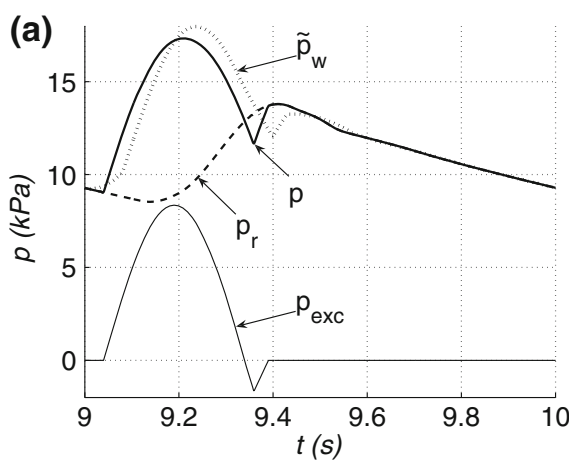

(c)

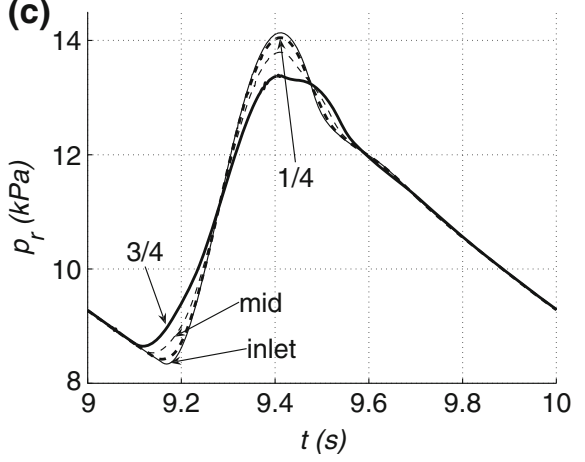

Fig. 4 a New excess pressure $\widetilde{p_{\text {exc }}}$ with time at $x=0$ (inlet), $l / 4(1 / 4), l / 2$ (mid) and 3l/4 (3/4) in the single-vessel aortic model. b Flow with time at the inlet $\left(q_{I N}\right)$ and midpoint (mid), and outflows $\widetilde{q}_{w}$ and $q_{C}$ driven by $\widetilde{p}_{w}$ and $p_{C}$, respectively
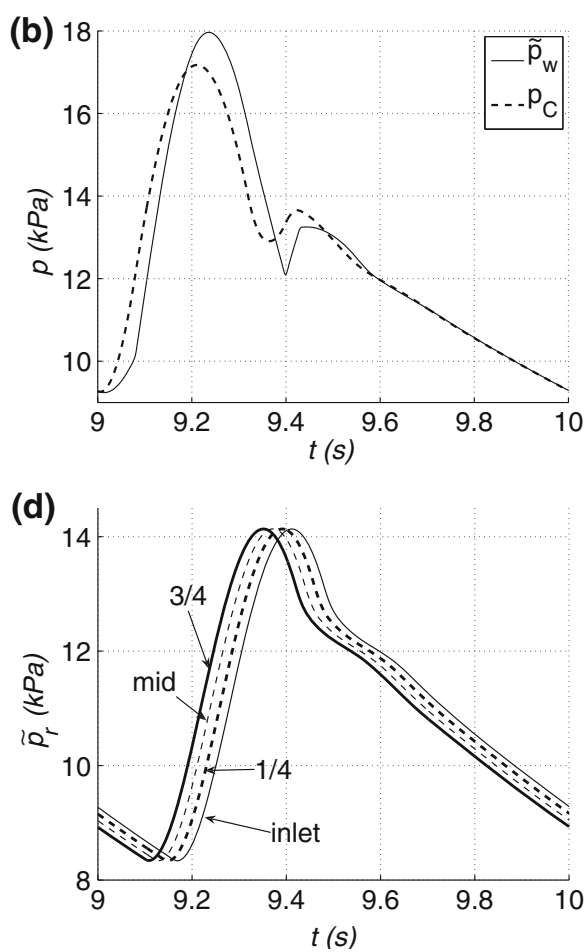

(d)

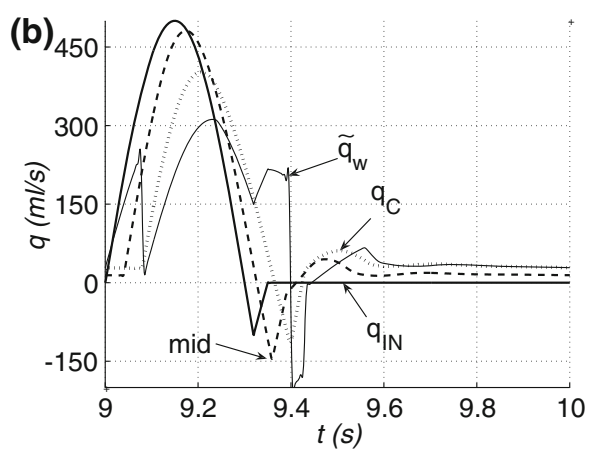

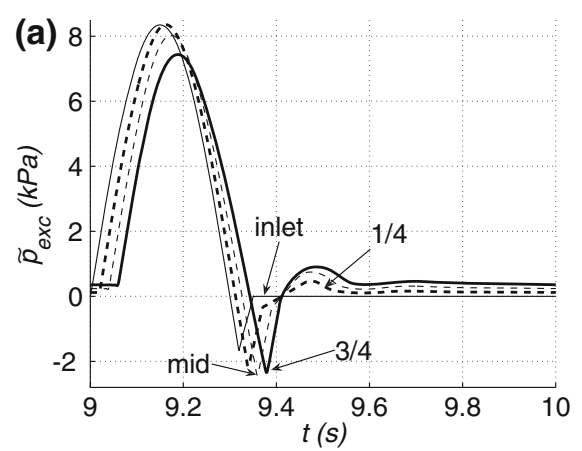

Figure 11 compares the diastolic decays of $\widetilde{p}_{w}$ in all the models studied using a semi-logarithmic plot. They are all approximately exponential with a time constant that differs from $R_{T} C_{T}=1.6 \mathrm{~s}$ by less than $5 \%$. If peripheral compliances are zero (i.e. $C_{j}=0, j=2, \ldots, M$ ) in the normal 55 -artery model and $C_{0 D}^{i}, i=1, \ldots, N$, are increased so that $C_{c}$ is equal to the original $C_{T}$, then the time constant is $1.6 \mathrm{~s}$. This is in agreement with Eq. 25 . In all cases, $\widetilde{p}_{w}$ approaches the asymptote $P_{\text {out }}=0$ with time.

\section{Discussion and Concluding Remarks}

This work has provided a theoretical and numerical description of the mechanics underlying the reservoir and excess pressures calculated using the two- and three-element windkessel models, and has proposed a new definition of them that provides valuable information for pulse wave analysis and overcomes the limitations of the current algorithms. Indeed, the two-element windkessel model requires a measurement of the left ventricular flow ejection $q_{I N}$, which cannot always be measured in vivo, to calculate the reservoir pressure. The three-element windkessel model does not require $q_{I N}$, but the reservoir pressure that provides is not defined explicitly.

We start by discussing the theoretical results obtained when the two-element windkessel model is used (e.g. Wang et al. 2003; Tyberg et al. 2008, 2009; Davies et al. 2010). In a network with inviscid flow and terminal outflows modelled using single resistances (i.e. all the compliance of the vessel walls is located within the 1-D model network and all the flow resistance is at the periphery of the network), the reservoir pressure $p_{w}$ calculated using Frank's windkessel Eq. 1 is the space-independent pressure to which blood pressure $p$ at any location tends with the increasing time in diastole. This result is in agreement with 
Fig. 5 Forward $\left(p_{f}\right.$ and $\left.q_{f}\right)$ and backward $\left(p_{b}\right.$ and $q_{b}$ ) contributions to a pressure $p$ and b flow $q$, and $\mathbf{c}$ new reservoir $\widetilde{p_{r}}$ and excess $\widetilde{p_{\text {exc }}}$ pressures with time in the midpoint of the single-vessel aortic model
Fig. 6 Pressure with time at the root of the ascending aorta (Edge 1, Asc) and the midpoint of the thoracic aorta II (Edge 33, Tho), left brachial (Edge 45, Bra) and right external iliac (Edge 52, Ili) arteries of the a normal and $\mathbf{b}$ well-matched 55-artery models. Pressures $\widetilde{p}_{w}$ in thick solid lines. After $t=10 \mathrm{~s}, q_{I N}=0$ to show the relaxation of both models

Fig. 7 Pressure (Tho) with time in the midpoint of the thoracic aorta II (Edge 33) and reservoir $p_{r}$ (with $\lambda=Z_{A o}$ ), peripheral $p_{p e r}$, excess $p_{\text {exc }}$ and conduit $p_{\text {con }}$ pressures at the same location in the a normal and b well-matched 55-artery models
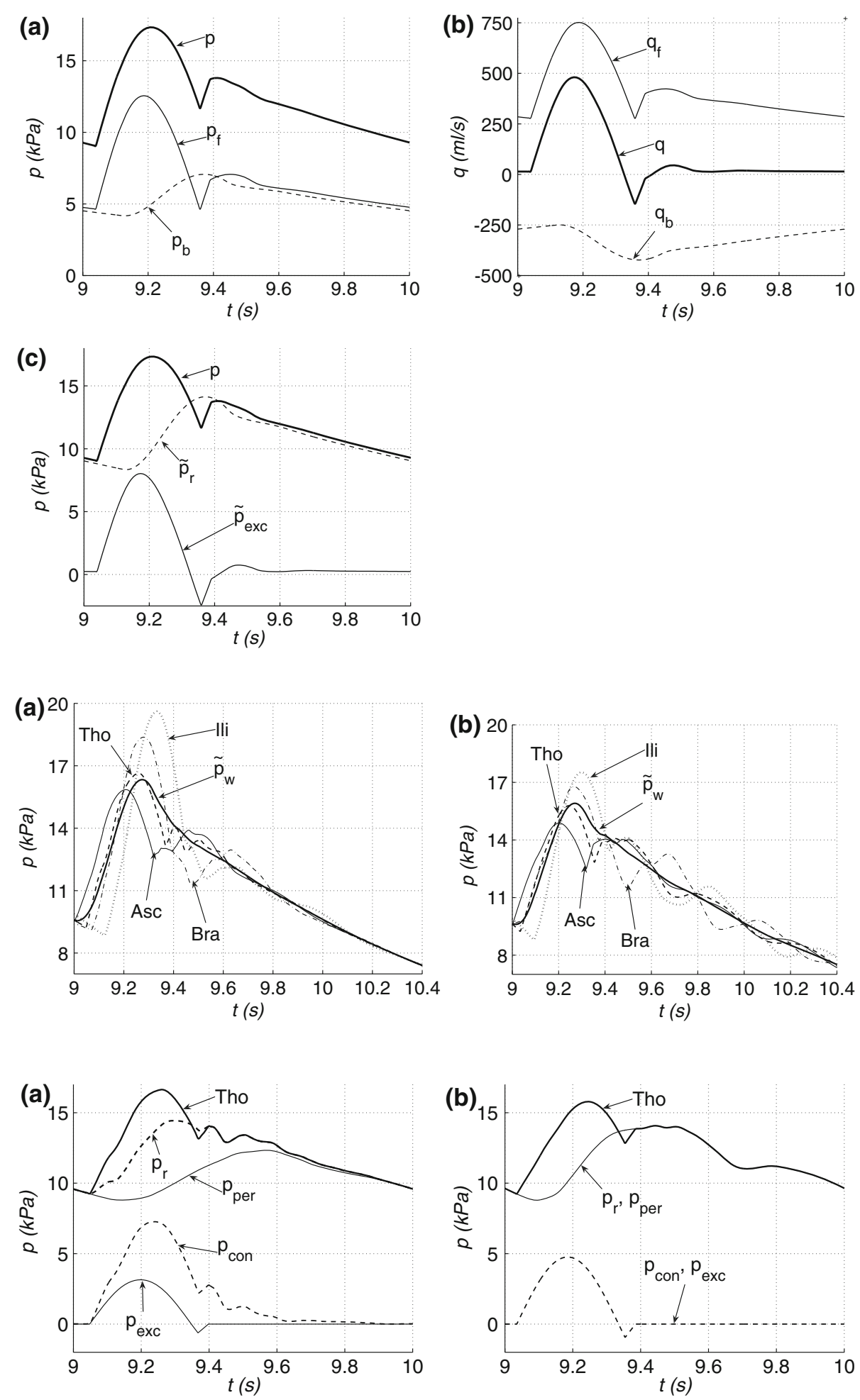

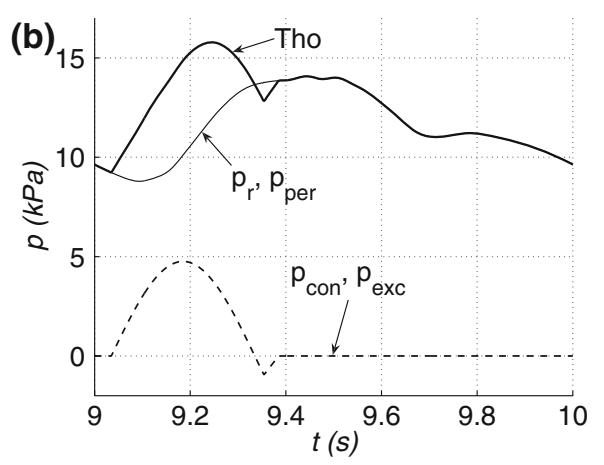

in vivo canine pressures, measured every $2 \mathrm{~cm}$ from the aortic root to the femoral artery, being approximately uniform during the last two-thirds of diastole (Wang et al. 2003). If we numerically allow for some peripheral compliance, then $p$ at any location tends to the space-independent $\widetilde{p}_{w}$ (Fig. 6), which like $p_{w}$ depends on global properties (left ventricular flow ejection, total compliance and resistance and outflow pressure) (Eq. 17), dictates the total flow to the periphery (Eq. 16) and is equal to $p_{w}$ when terminal outflows are modelled using single resistances.

Since $\widetilde{p}_{w}$ was derived assuming blood inertia to be zero, the results in the previous paragraph suggest that there is a 
Fig. 8 a Peripheral pressure $p_{p e r}$ with time at the root of the ascending aorta (Edge 1, Asc), and the midpoint of the thoracic aorta II (Edge 33, Tho), left brachial (Edge 45, Bra) and right external iliac (Edge 52, Ili) arteries of the well-matched 55artery model. b New reservoir pressure $\widetilde{p_{r}}$ with time at $x=0$ (inlet), $l / 4$ (1/4), $l / 2$ (mid) and $3 l / 4(3 / 4)$ in the left brachial artery of the normal 55-artery model. c $\widetilde{p_{r}}$ with time at Asc, Tho, Bra and Ili of the normal 55-artery model. After $t=10$ $\mathrm{s}, q_{I N}=0$ in panels (a) and (c)
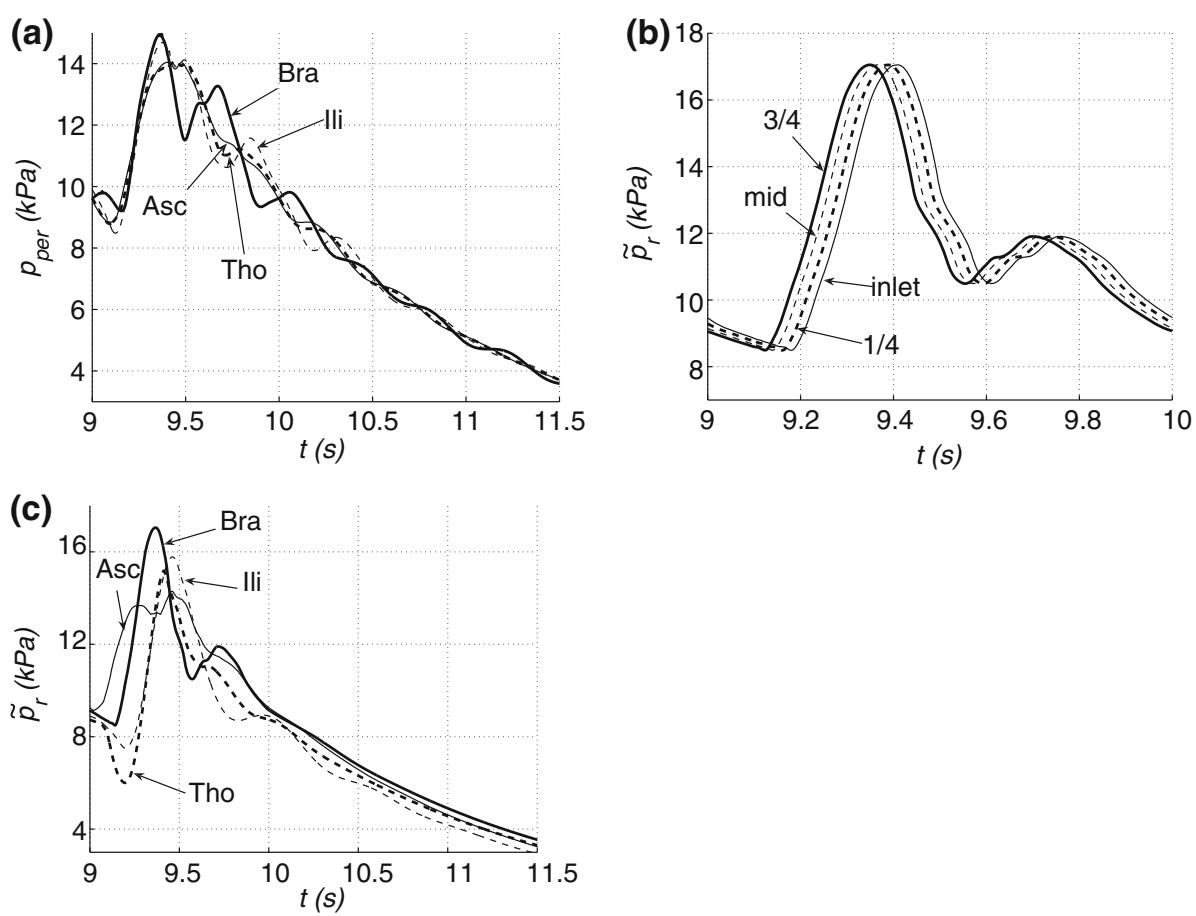

Fig. 9 a, b Windkessel $\widetilde{p}_{w}$ and compliance-weighted spaceaverage $p_{C}$ pressures, and $\mathbf{c}, \mathbf{d}$ outflows $\widetilde{q}_{w}$ and $q_{C}$ driven by $\widetilde{p}_{w}$ and $p_{C}$, respectively, with time in the normal $(\mathbf{a}, \mathbf{c})$ and well-matched (b, d) 55-artery models
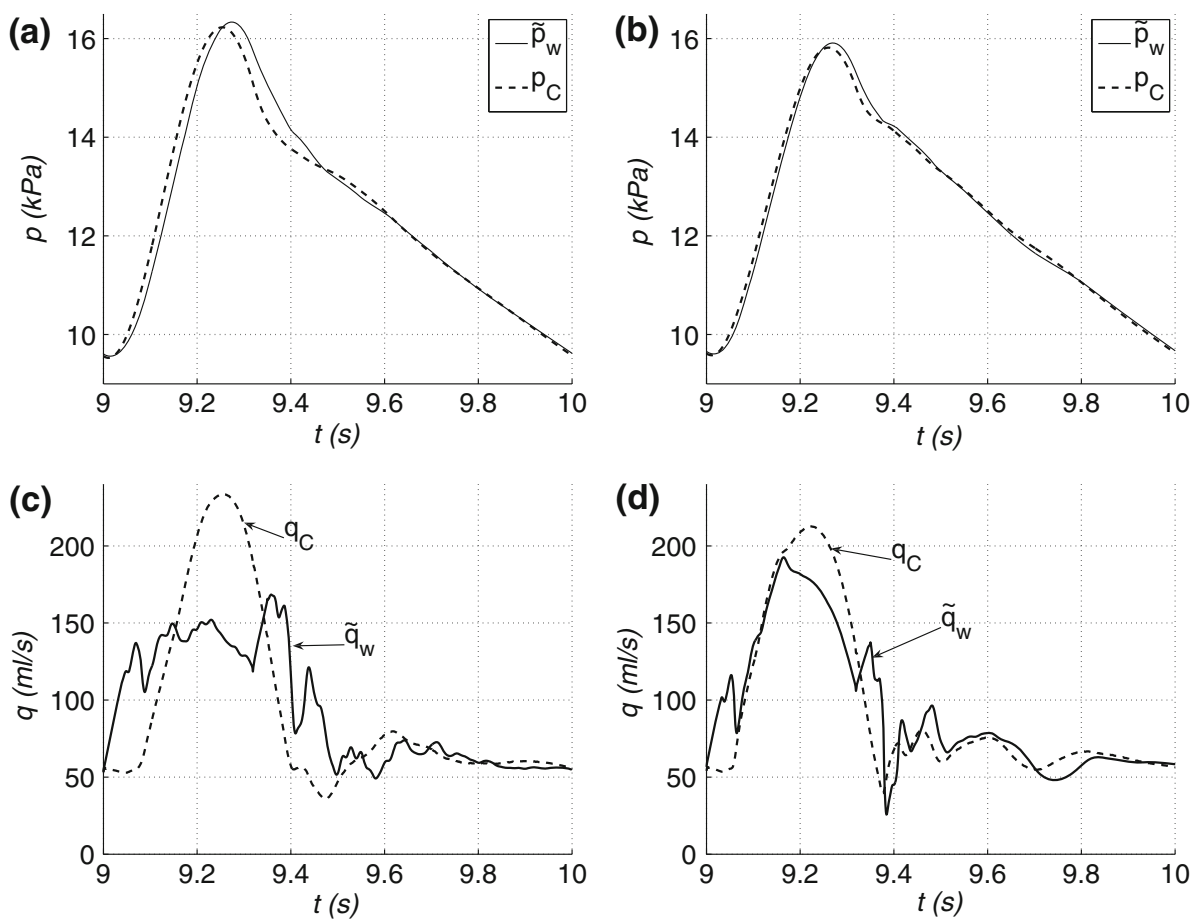

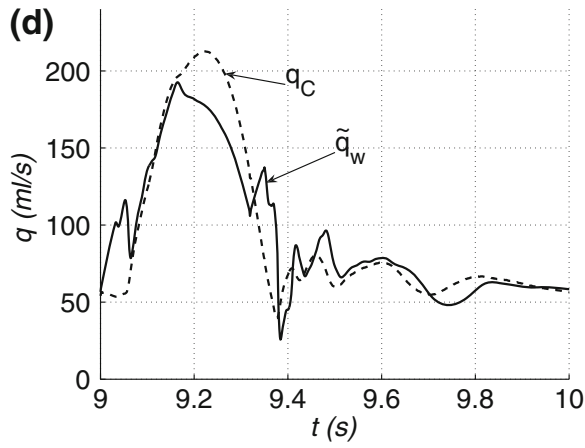

progressive loss of blood inertia in diastole, which is recovered by the time of the next left ventricular ejection. Thus, a clear distinction exists between the systolic and diastolic mechanics underlying blood flow in systemic arteries. In systole, the changes in blood pressure and flow produced by the contraction of the left ventricle propagate in the form of waves (called pulse waves) through the systemic arteries. These waves are reflected at the arterial junctions, peripheral branches, aortic valve (when shut), and any other change in arterial geometry and elasticity, and produce space-dependent pressures and flows. With the increasing time, the number of reflected waves increases exponentially, but the amplitude of the pressure and flow changes associated with these waves tends to decrease 
Fig. 10 Forward $\left(p_{f}\right.$ and $\left.q_{f}\right)$ and backward $\left(p_{b}\right.$ and $\left.q_{b}\right)$ contributions to a pressure $p$ and b flow $q$, and $\mathbf{c}$ new reservoir $\widetilde{p}_{r}$ and excess $\widetilde{p_{\text {exc }}}$ pressures with time in the midpoint of the thoracic aorta II (Edge 33) of the normal 55-artery model
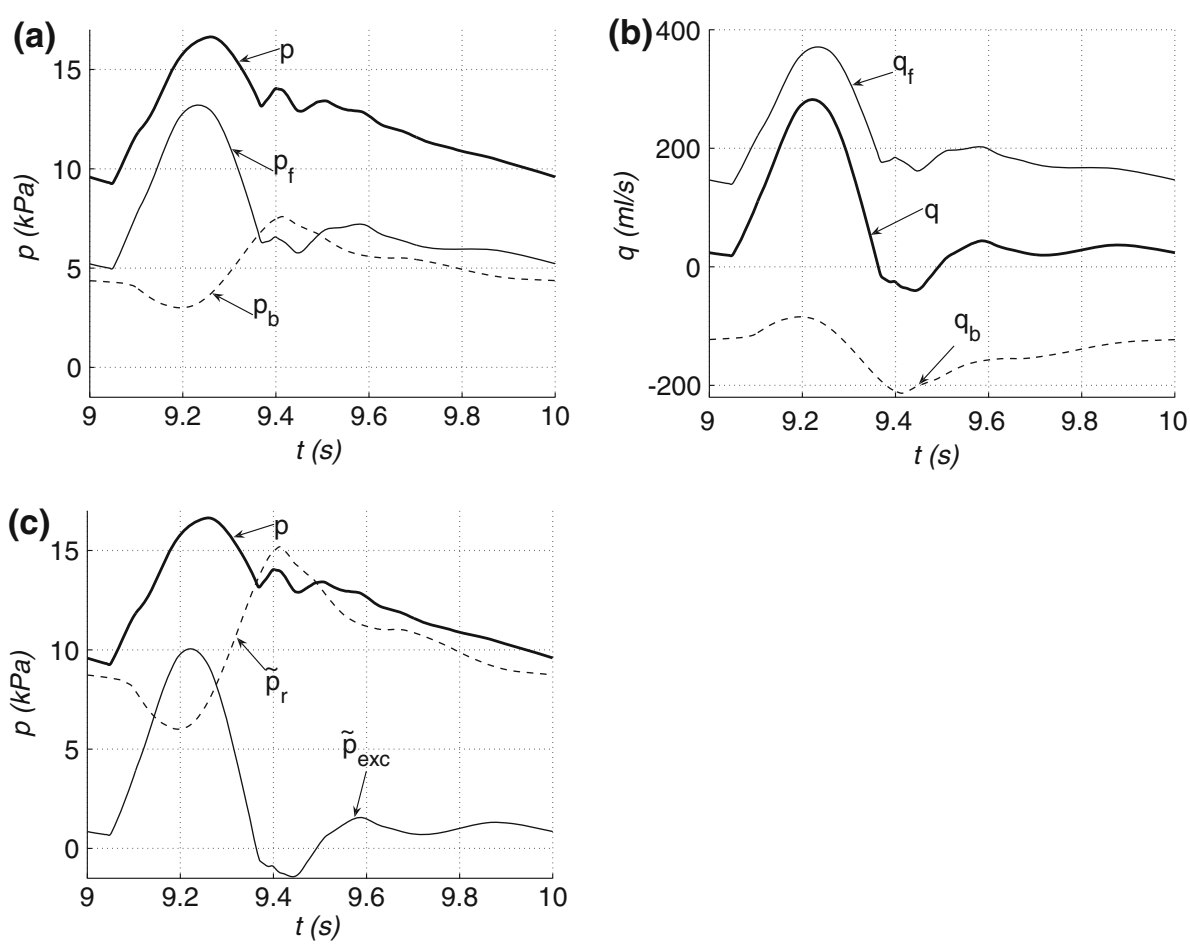

exponentially (Alastruey et al., 2009). In diastole, waves progressively lose inertia and the flow is dominated by the global relaxation of the compliant vessels.

The numerical results show a transient exponential building-up in the diastolic pressure waveform $p$ from the initial zero pressure. Separation of $p$ into peripheral $p_{p e r}$ and conduit $p_{c o n}$ contributions suggests that this buildingup is due to $p_{p e r}$ and, hence, generated by waves originated as reflections at the peripheral outflows (Alastruey et al., 2009). Indeed, a simulation started at zero pressure in a network with completely absorbent peripheral outflows produces a periodic pressure $p=p_{\text {con }}$ without a physiological diastolic decay from the first heartbeat. Therefore, peripheral resistances must be greater than peripheral characteristic impedances in order for arteries to store sufficient blood volume and potential energy in systole and generate sufficient pressure and kinetic energy for the perfusion of the periphery in diastole.

The numerical results in this work also suggest that $\widetilde{p}_{w}$ in systole and early diastole is well approximated by the compliance-weighted space-average pressure of the arterial network $p_{C}$ defined in 14. In diastole $\widetilde{p}_{w}$ and $p_{C}$ (and the flows $\widetilde{q}_{w}$ and $q_{C}$ that they drive) gradually converge to the same shape (Figs. 3b, 4b, 9), which is consistent with the gradual convergence of $p$ to $\widetilde{p}_{w}$. Therefore, $p_{C}$ provides an approximate physical interpretation of $\widetilde{p}_{w}$ (and hence $p_{w}$ ), and shows that the more compliant arteries are, the larger their contribution is to the total peripheral outflow in diastole.
The mechanics underlying the reservoir pressure $p_{r}$ calculated using the three-element windkessel model (e.g. Aguado-Sierra et al. (2008); Davies et al. (2007); Parker (2009); Vermeersch et al. (2009)) have been studied taking $\lambda=Z_{A o}$. Under this assumption, this work has shown that $p_{r}$ is not space-independent in systole and early diastole (Figs. 3c, 8a). Moreover, the mean $p_{r}$ is smaller than the mean $p_{w}$ when the flow is periodic; the mean $p_{w}$ being equal to the mean blood pressure for inviscid flow. AguadoSierra et al. (2008) assumed well-matched junctions for forward-travelling waves, which seems to be a reasonable approximation in normal humans and animals (Milnor, 1989; Papageorgiou and Jones, 1987). Under this assumption, this work has shown that $p_{r}$ is equal to the pressure $p_{p e r}$ generated by the reflected waves originated at the terminal reflection sites, and $p_{e x c}$ is the pressure $p_{c o n}$ obtained by propagating the left ventricular flow ejection without any peripheral reflection (Fig. 7b). The larger the reflection coefficients for forward-travelling waves at junctions are, the less valid these descriptions of $p_{r}$ and $p_{\text {exc }}$ become (Fig. 7a). Indeed, if junctions are not well-matched for forward-travelling waves, $p_{r}$ calculated at the ascending aorta with $\lambda=Z_{A o}$ differs from $p_{p e r}$ in systole and early diastole, and only converges to $p_{p e r}$ later in diastole. A similar relation, therefore, exists between $p_{e x c}=p-p_{r}$ and $p_{\text {con }}=p-p_{\text {per }}$.

The new reservoir $\widetilde{p_{r}}=p-\rho c q / A_{0}$ and excess $\widetilde{p_{\text {exc }}}=$ $\rho c q / A_{0}$ pressures (Eq. 26) proposed here overcome the limitations of the two- and three-element windkessel 
models, since $\widetilde{p_{r}}$ and $\widetilde{p_{\text {exc }}}$ are independent of $q_{I N}$ and well defined at any location in the arterial network. Moreover, if local compliances are constant within each arterial edge, $\widetilde{p_{r}}$ is space-independent within each edge (except for the time alignment) unlike $p_{r}$, and $\widetilde{p_{e x c}}$ is always proportional to the local flow unlike $p_{\text {exc }}$. Calculation of $\widetilde{p_{r}}$ requires simultaneous pressure and velocity $q / A_{0}$ measures and an estimation of the local pulse wave speed $c$, which can be obtained using the methods in Davies et al. (2006) and Khir et al. (2001).

In the models studied here, $\widetilde{p_{r}}$ is the major contribution to the diastolic pressure decay, while $\widetilde{p_{\text {exc }}}$ plays a more significant role in shaping the pressure waveform in systole and early diastole (Figs. 5c, 10c). At the aortic root $\widetilde{p_{r}}$ produces all the diastolic pressure decay, since $\widetilde{p_{e x c}}=0$ in diastole due to $q=0$. Note that $\widetilde{p_{r}}$ is made up of contributions to pressure from distal locations (Eq. 27) and, hence, plays a similar role to $p_{p e r}$. However, $p_{p e r}$ accounts only for distal contributions that originate at peripheral reflection sites, whereas $\widetilde{p_{r}}$ includes contributions from reflections at any other change in distal arterial geometry or elasticity; e.g. at all the arterial junctions.

The separation of $p$ into $\widetilde{p_{r}}$ and $\widetilde{p_{\text {exc }}}$ is closely related to the separation of $p$ into forward $p_{f}$ and backward $p_{b}$ waves described in Westerhof et al. (1972), Parker and Jones (1990), and Hughes and Parker (2009), as shown by Eq. 27. In diastole, $p_{f}$ and $p_{b}$ are approximately equal and relatively large, leading to self-cancelling forward $q_{f}$ and backward $q_{b}$ flow waves (Figs. 5a, b, 10a, b). This diastolic pattern was considered to be a necessary result of the wave separation analysis, but its origin was difficult to explain physically, since the heart is not ejecting blood into the aorta in diastole (Parker 2009).

Pulse wave analysis using $\widetilde{p_{r}}$ and $\widetilde{p_{\text {exc }}}$ provides a more physically plausible description of $p_{f}$ and $p_{b}$ and their relation to $q$. At any time, $\widetilde{p_{\text {exc }}}=p_{f}-p_{b}$ is proportional to $q$ and, hence, determines the magnitude and direction of $q$ (Eq. 27). The increase in systolic $p_{f}$ at the aortic root generated by the left ventricular flow ejection is propagated to distal locations increasing $\widetilde{p_{\text {exc }}}$. In diastole, a relatively smaller $\widetilde{p_{\text {exc }}}$ indicates a better balance between proximal and distal contributions to $p$. In normal diastolic conditions, $q_{I N}=0$ and hence $p_{b}$ is totally reflected at the closed aortic valve, so that $p_{f}=p_{b}$. Thus $\widetilde{p_{r}}=2 p_{b}$ is a better approximation to the diastolic pressure decay than $p_{b}$ is. On the other hand, $p_{f}$ is partially reflected at the peripheral reflection sites. This pattern of wave reflections of $p_{f}$ at the peripheral reflection sites and $p_{b}$ at the closed aortic valve leads to a positive $\widetilde{p_{\text {exc }}}$ in most of diastole, so that $q$ is mostly directed toward the periphery. The progressive loss of diastolic blood inertia in the 1-D balance of momentum that leads to the convergence of $p$ to $\widetilde{p}_{w}$ also yields uniform $p_{f}$ and $p_{b}$ in diastole, and hence $\widetilde{p_{r}}$, except for the time

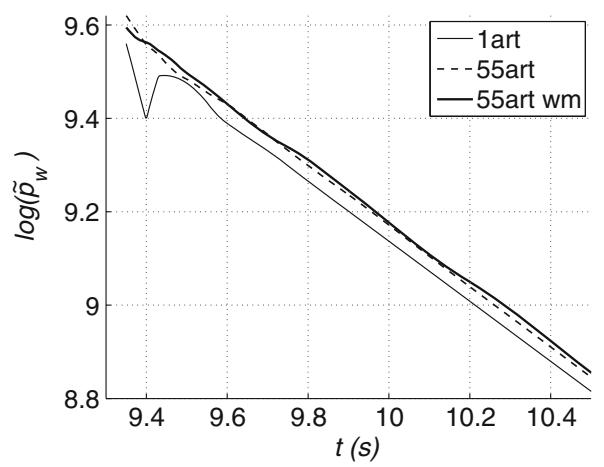

Fig. 11 Diastolic $\widetilde{p}_{w}$ on a logarithm scale with time in the singlevessel aorta (1art) and the normal (55art) and well-matched (55art wm) 55 -artery models. After $t=10 \mathrm{~s}, q_{I N}=0$ to show the relaxation of the models

alignment (Fig. 8c). The summation of $p_{f}$ and $p_{b}$ in diastole produces a space-independent $p$ and their difference leads to non-zero $q$ in diastole.

The numerical results presented here have shown that the time constant that characterises the diastolic pressure decay can be reasonably estimated using an exponential fitting to diastolic pressure at an arbitrary arterial location (Fig. 11). It is important to emphasise that the longer the diastolic time (e.g. when a heartbeat is missed) the better the accuracy of the fitting. However, the assumption of constant total compliance becomes less valid for a larger change in pressure, since in vivo arterial compliance is pressure dependent (Roy 1880-1882; Frank 1899).

Experimental and clinical investigations will be required to assess the validity of the results presented here and test the relevance of the new definition of reservoir and excess pressures. According to this work, they should improve our understanding of the relationship between the pattern of pulse waveforms and the physical properties of the cardiovascular system.

Acknowledgments The author would like to acknowledge Emeritus Professor Kim Parker (Imperial College London) for his helpful comments on the paper. This work was funded by a British Heart Foundation Intermediate Basic Science Research Fellowship (FS/09/ 030/27812)

Open Access This article is distributed under the terms of the Creative Commons Attribution Noncommercial License which permits any noncommercial use, distribution, and reproduction in any medium, provided the original author(s) and source are credited.

\section{References}

Aguado-Sierra J, Alastruey J, Wang J-J, Hadjiloizou N, Davies J, Parker K. Separation of the reservoir and wave pressure and velocity from measurements at an arbitrary location in arteries. Proc Inst Mech Eng Part H J Eng Med. 2008;222:403-16. 
Alastruey J. Numerical modelling of pulse wave propagation in the cardiovascular system: development, validation and clinical applications. Ph.D. thesis, Imperial College London, University of London, UK 2006.

Alastruey J, Parker K, Peiró J, Sherwin S. Lumped parameter outflow models for 1-D blood flow simulations: effect on pulse waves and parameter estimation. Commun Comput Phys. 2008;4: 317-36.

Alastruey J, Parker K, Peiró J, Sherwin S. Analysing the pattern of pulse waves in arterial networks: a time-domain study. J Eng Math. 2009;64:331-51.

Caro C, Pedley T, Schroter R, Seed W. The mechanics of the circulation. Oxford: Oxford University Press; 1978.

Davies J, Whinnett Z, Francis D, Willson K, Foale R, Malik I, Hughes A, Parker K, Mayet J. Use of simultaneous pressure and velocity measurements to estimate arterial wave speed at a single site in humans. Am J Physiol Heart Circ Physiol. 2006;290:H878-85.

Davies J, Hadjiloizou N, Leibovich D, Malaweera A, Alastruey J, Whinnett Z, Manisty C, Francis D, Aguado-Sierra J, Foale R, Malik I, Parker K, Mayet J, Hughes A. Importance of the aortic reservoir in determining the shape of the arterial pressure waveform-The forgotten lessons of Frank. Artery Res. 2007;1: $40-5$.

Davies J, Baksi J, Francis D, Hadjiloizou N, Whinnett Z, Manisty C, Aguado-Sierra J, Foale R, Malik I, Tyberg J, Parker K, Mayet J, Hughes A. The arterial reservoir pressure increases with aging and is the major determinant of the aortic augmentation index. Am J Physiol Heart Circ Physiol. 2010;298:H580-6.

Frank O. Die Grundform des arteriellen Pulses. Erste AbhandlungMathematische Analyse Z Biol. 1899;37:483-526. (Translated by Sagawa K, Lie RK, Schaefer J. J Mol Cell Cardiol. 1990;22:253-77).

Hughes A, Parker K. Forward and backward waves in the arterial system: impedance or wave intensity analysis?. Med Bio Eng Comput. 2009;47:207-10.

Khir A, O'Brien A, Gibbs J, Parker K. Determination of wave speed and wave separation in the arteries. J Biomech. 2001;34:1145-55.

Matthys K, Alastruey J, Peiró J, Khir A, Segers P, Verdonck P, Parker $\mathrm{K}$, Sherwin S. Pulse wave propagation in a model human arterial network: assessment of 1-D numerical simulations against in vitro measurements. J Biomech. 2007;40:3476-86.

Milnor W. Hemodynamics. Baltimore: Williams and Wilkins; 1989.

Olufsen M, Peskin C, Kim W, Pedersen E, Nadim A, Larsen J. Numerical simulation and experimental validation of blood flow in arteries with structured-tree outflow conditions. Annals Biomed Eng. 2000;28:1281-99.
Papageorgiou G, Jones N. Arterial system configuration and wave reflection. J Biomed Eng. 1987;9:299-301.

Parker K, Jones C. Forward and backward running waves in the arteries: analysis using the method of characteristics. J Biomech Eng. 1990;112:322-6.

Parker K. An introduction to wave intensity analysis. Med Bio Eng Comput. 2009;47:175-88.

Quarteroni A, Formaggia L. Mathematical modelling and numerical simulation of the cardiovascular system. In Ayache N, editors. Modelling of living systems. Amsterdam: Elsevier; 2004.

Roy C. The elastic properties of the arterial wall. J Physiol (Lond). $1880-1882 ; 3: 125-59$.

Steele B, Wan J, Ku J, Hughes T, Taylor C. In vivo validation of a one-dimensional finite-element method for predicting blood flow in cardiovascular bypass grafts. IEEE Trans Biomed Eng. 2003;50:649-56.

Stergiopulos N, Young D, Rogge T. Computer simulation of arterial flow with applications to arterial and aortic stenoses. J Biomech. 1992;25:1477-88.

Tyberg J, Shrive N, Bouwmeester J, Parker K, Wang J-J. The reservoir-wave paradigm: potential implications for hypertension. Curr Hypertens Rep. 2008;4:203-12.

Tyberg J, Davies J, Wang Z, Whitelaw W, Flewitt J, Shrive N, Francis D, Hughes A, Parker K, Wang J-J. Wave intensity analysis and the development of the reservoir-wave approach. Med Biol Eng Comput. 2009;47:221-32.

Čanić S, Kim E. Mathematical analysis of the quasilinear effects in a hyperbolic model of blood flow through compliant axi-symmetric vessels. Math Meth Appl Sci. 2003;26:1161-86.

Vermeersch SJ, Rietzschel E, Buyzere MD, Bortel LV, Gillebert T, Verdonck P, Segers P. The reservoir pressure concept: the 3element windkessel model revisited? Application to the Asklepios population study. J Eng Math. 2009;64:417-428

Wang J-J, O'Brien A, Shrive N, Parker K, Tyberg J. Time-domain representation of ventricular-arterial coupling as a windkessel and wave system. Am J Physiol Heart Circ Physiol. 2003;284: H1358-68.

Westerhof N, Bosman F, de Vries C, Noordergraaf A. Analog studies of the human systemic arterial tree. J Biomech. 1969;2:121-43.

Westerhof N, Elzinga G, Sipkema P. An artificial arterial system for pumping hearts. J Appl Physiol. 1971;31:776-81.

Westerhof N, Sipkema P, Bos GVD, Elzinga G. Forward and backward waves in the arterial system. Cardiov Res. 1972;6: 648-56.

Zhang H, Li JK-J. A novel wave reflection model of the human arterial system. Cardiovasc Eng. 2009;9:39-48. 